\title{
Effect of Exercise Prior or After Ischemia on the Density of Neurons and Astrocytes in the Brain of Rats
}

\author{
${ }^{1}$ Laila Cristina Moreira Damázio, ${ }^{1}$ Raphael Teixeira Rocha Melo, ${ }^{2}$ Maira de Castro Lima, ${ }^{2}$ Hélio Batista dos \\ Santos, ${ }^{2}$ Rosy Iara Maciel de Azambuja Ribeiro, ${ }^{2}$ Natália Ribeiro Alves, ${ }^{3}$ Betânia Souza Monteiro, ${ }^{4}$ Antônio \\ José Natali, ${ }^{3}$ Ricardo Junqueira Del Carlo and ${ }^{1}$ Izabel Regina dos Santos Costa Maldonado
}

${ }^{1}$ Graduate Program in Cellular and Structural Biology, Federal University of Viçosa, 36570-000, Viçosa, MG, Brazil

${ }^{2}$ Group of Morphology, Federal University of São João Del Rei-CCO, 35501-296, Divinópolis, MG, Brazil

${ }^{3}$ Department of Veterinary Medicine, Federal University of Viçosa, 36570-000, Viçosa, MG, Brazil

${ }^{4}$ Department of Physical Education, Federal University of Viçosa, 36570-000, Viçosa, MG, Brazil

Article history

Received: 11-06-2014

Revised: 12-06-2014

Accepted: 27-10-2014

Corresponding Author:

Laila Cristina Moreira Damázio,

Graduate Program in Cellular and

Structural Biology, Federal

University of Viçosa, 36570-000,

Viçosa, MG, Brazil

Email: lailacmdamazio@gmail.com

\begin{abstract}
The aim of this study was to analyse the density of astrocytes and neurons in the motor areas of the cortex, striatum and hippocampus of rats that performed exercise before or after cerebral ischemia. A total of 36 Wistar rats were separated into two groups: One that was subjected to Middle Cerebral Artery Occlusion (MCAO) and the other that underwent surgical access in the absence of ischemia. Both groups were subdivided into: Animals that underwent exercise prior to MCAO and sham surgery (AI and AC, $n=12$ ); animals that underwent exercise after MCAO and sham surgery (DI and DC, $n$ $=12$ ); and animals that did not undergo exercise (SI and SC, $n=12$ ). The brains of mice were sectioned and stained by the Nissl method for staining of neurons and the astrocytes were labeled by immunohistochemistry with glial fibrillary acidic protein. Physical training was conducted after the surgery for 6 weeks (30 min/day), 5 days per week. The animals exercised before cerebral ischemia showed greater density of neurons in the striatum, in the apex of the dentate gyrus of the hippocampus, in the motor cortex contra lateral to the lesion and more astrocytes in the motor cortex of the cerebral hemispheres compared with the untrained group $(p<0.05)$. The density of neurons in the brain regions analysed remained unchanged in exercised animals from the fourth day after cerebral ischemia when compared with the sedentary group.
\end{abstract}

Keywords: Cerebral Ischemia, Immunohistochemistry for Glial Fibrillary Acidic Protein, Astrocytes, Neurons

\section{Introduction}

Astrocytes are involved in neuroplasticity through the absorption and release of brain-derived neurotrophic factor, the synthesis of Glial Fibrillary Acidic Protein (GFAP) and the transport of glutamate and glutamine synthetase (Bergami et al., 2008; Eng et al., 2000). A mechanism for the regulation of excitotoxicity in cerebral ischemia is the conversion of glutamate to glutamine by the enzyme glutamine synthetase, present in astrocytes (Cammer et al., 1990; Eddleston and Mucke, 1993).

The most abundant glial cells in the central nervous system (Abbott, 2002; Goudstein and Betz, 1986) are the astrocytes, covering $90 \%$ of the cerebrovascular surface (Willis et al., 2004). These cells participate in the regulation of free neurotransmitters, immune response, repair of the extracellular matrix, control of the cerebrovascular barrier and trophic support (Eddleston and Mucke, 1993). GFAP is a protein that makes up the intermediate filaments of the cytoskeleton of astrocytes (Eng et al., 2000) and its immunostaining allows us to highlight the reactive astrocytes in central nervous system lesions (Ridet et al., 1997).

A high density of astrocytes plays a part in a mechanism related to the recovery of the area around the site of cerebral ischemia in rats trained after ischemia ( $\mathrm{Li}$ et al., 2005), since these cells also participate in the regulation of the blood-brain barrier and are important in maintaining brain permeability (Arthur et al., 1987; Kondo et al., 1996; Tao et al., 1987).

Exercise reduces brain injury by decreasing local edema and by the maintenance of micro vascular integrity (Ding et al., 2006; Li et al., 2005). It also promotes the remodeling of synapses in the hippocampus (Liu et al., 
2011). Astrocytes play an important role in this process (Humm et al., 1998; Taskapiliogly et al., 2009) by synthesizing glial-derived neurotrophic factor, which is responsible for the increased survival of dopaminergic neurons and by promoting neuroplasticity (Senna et al., 2011; Henderson et al., 1994; Lin et al., 1993).

As there are few studies that describe the density of astrocytes and neurons in rats with cerebral ischemia undergoing physical exercise, this study aimed to analyse the density of astrocytes and neurons in the brain of rats that performed exercise before or after brain ischemia.

\section{Materials And Methods}

\section{Animals}

A total of 36 Wistar rats were obtained from the animal colony of the Federal University of Viçosa. Their mean weight was $138.7 \pm 2.18 \mathrm{~g}$ and for 30 days they were kept in cages with free access to water and food (12-h photoperiod at room temperature between 21 and $22^{\circ} \mathrm{C}$ and relative humidity of $60-70 \%$ ). The animals were weighed at the beginning of the experiment (30 days), immediately before surgery (76 days) and after surgery ( 81 days) and at the end of the experiment (126 days). All procedures were approved by the Ethics Committee on Animal Use, Federal University of Viçosa (protocol 32/2011).

A total of 18 animals were subjected to ischemia by transient Middle Cerebral Artery Occlusion (MCAO) and distributed (Table 1) in the following subgroups: AI ( $n=6$ ) underwent exercise on the treadmill (Insight Instruments $^{\circledR}$, Ribeirão Preto, SP) prior to the ischemia; DI $(n=6)$ underwent exercise on the treadmill after ischemia; and SI $(n=6)$ did not undergo exercise.

Another 18 animals (control) underwent surgical access, but not to MCAO and were distributed (Table 1) in the following subgroups: AC $(n=6)$ were trained before surgery; $\mathrm{AD}(n=6)$ were trained after surgery; and $\mathrm{SC}(n=6)$ were not trained on the mat.

\section{Physical Training}

Animals that were exercised before the surgical procedure were adapted to the treadmill for 5 days at a speed of $10-12 \mathrm{~m} / \mathrm{min}$, for $12 \mathrm{~min} /$ day. After the adaptation period, the rats belonging to the groups trained before surgery (AI, AC) were subjected to the physical training treadmill without inclination for 6 weeks, 5 days a week for $30 \mathrm{~min} /$ day at a speed of 15 $\mathrm{m} / \mathrm{min}$. The speed of the exercise was gradually increased, from $10 \mathrm{~m} / \mathrm{min}$ on the first day, to $12 \mathrm{~m} / \mathrm{min}$ on the second day and from the third day onwards 15 $\mathrm{m} / \mathrm{min}$ (Ding et al., 2004b; 2006).

The rats allocated to the exercise groups after surgery (DI and DC) began the exercises 7 days after the surgical procedure and the adjustment period on the treadmill.
The exercises after MCAO (DI) and after the sham operation (DC) lasted 6 weeks and the rats exercised for $30 \mathrm{~min} /$ day, on the treadmill without inclination, 5 days per week, with a speed of $12 \mathrm{~m} / \mathrm{min}$ (Liu et al., 2011; Zhang et al. 2012a; 2012b). The exercise speed was gradually increased: On the first day the rats ran with a speed $8 \mathrm{~m} / \mathrm{min}$, on the second day at $10 \mathrm{~m} / \mathrm{min}$ and on the third day at $12 \mathrm{~m} / \mathrm{min}$ (Liu et al., 2011; Zhang et al. $2012 \mathrm{a} ; 2012 \mathrm{~b}$ ). The rats that were able to run for $2 \mathrm{~min}$ at speeds of 8,11 and $12 \mathrm{~m} / \mathrm{min}$ in the first 3 days of the physical training continued in the research.

\section{Occlusion of the Middle Cerebral Artery}

The animals were pre-treated with the analgesic fentanyl $(0.3 \mathrm{mg} / \mathrm{kg}$ intramuscularly $)$, sedated with diazepam $(2.5 \mathrm{mg} / \mathrm{kg}$ intraperitoneally) and anesthetized with an anesthetic circuit of isoflurane with 100\% oxygen. Prophylaxis was performed with enrofloxacin $(10 \mathrm{mg} / \mathrm{kg})$ intramuscularly.

After anesthesia, saline atropine diluted to $2 \%(0.1$ $\mathrm{mL}$ per animal) was administered intraperitoneally for the prevention of cardiac arrhythmia and bronchial hypersecretion secondary to mechanical stimulation of the vagal nerve during the operation.

The surgical procedure used to perform transient occlusion of the Middle Cerebral Artery (MCA) has been described by Longa et al. (1989). After trichotomy and antisepsis of the ventral cervical region, a median sagittal incision of approximately 3 $\mathrm{cm}$ in length was performed. The tissues were divulsed, exposing the bifurcation of the left Common Carotid Artery (CCA). The pterygopalatine branch of the Internal Carotid Artery (ICA) was clamped at its origin. The External Carotid Artery (ECA) was ligated with nylon Vicryl n-0 wire at the distal bifurcation of the CCA. After the CCA and ICA were clamped, the ECA was sectioned for passage of the monofilament (silicone, $0.12 \mathrm{~mm}$ in diameter and $5.0 \mathrm{~mm}$ long; Doccol $^{\circledR}$ ) and the wire occlusion. The wire was inserted through the left ACE within the ICA to the origin of the MCA, approximately at a $20-21 \mathrm{~mm}$ distance from the bifurcation of the CCA. To allow passage of the wire occlusion, the clip was removed from the ACI. To determine the length of the wires, they were previously marked at the lengths of 18,20 and $22 \mathrm{~mm}$. The introduction of $20 \mathrm{~mm}$ from the edge of the occlusion is associated with slight resistance to the passage of the wire, indicating the location of the origin of the ACM. The surgical incision was closed, keeping the wire of the occlusion and clip in the CCA.

The wire and the clip remained for $60 \mathrm{~min}$ (Duarte et al., 2003; Ma et al., 2006) then the animal was anesthetized again, the wire was removed and the proximal portion of the ACE to the bifurcation of the CCA (where the wire was introduced) was connected. 
Laila Cristina Moreira Damázio et al. / American Journal of Neuroscience 2014, 5 (2): 18.25 DOI: 10.3844/amjnsp.2014.18.25

Table 1. Distribution of rats $(n=36)$ according to experimental group

\begin{tabular}{llll}
\hline & Exercise before & Exercise after & Sedentary \\
\hline Animals subjected to cerebral ischemia & AI & DI \\
& $(n=6)$ & SI \\
Operated animals, but without cerebral ischemia & AC & $(n=6)$ & DC \\
& $(n=6)$ & $(n=6)$ & SC \\
\hline
\end{tabular}

Approximation of tissues and dermal suture were performed with Vicryl 3-0 nylon.

After surgery, the animals were kept in recovery for $2 \mathrm{~h}$ under warm conditions to maintain body temperature between 36 and $38^{\circ} \mathrm{C}$, with water and food. To reset body fluid, saline was injected intraperitoneally daily $(10 \mathrm{~mL})$ during the first 3 days of observation (Modo et al., 2000).

\section{Neurological Evaluation Scale}

The scale was used for the model of MCAO in rats proposed by Menzies et al. (1992). The scale has scores of 0-4; the higher the score, the worse the neurological deficit. On the first day after surgery, the animals with a score of 4 were included in the experiment and used in subgroups AI (exercise before MCAO), DI (exercise after MCAO) and SI (sedentary and MCAO).

\section{Histochemistry by the Nissl Method}

After 126 days, all animals were anesthetized with intraperitoneal injection of ketamine $(1 \%, 30 \mathrm{mg} / \mathrm{kg})$ and xylazine (4 mg/kg) (Lim et al., 2008). Subsequently, hearts were perfused with heparinized saline $(0.9 \% \mathrm{NaCl}$ containing $5000 \mathrm{IU}$ heparin/l) for $5 \mathrm{~min}$, followed by perfusion with a $4 \%$ solution of paraformaldehyde for 5 min. Then, brains were removed and sliced into $1 \mathrm{~mm}$ sections in the coronal plane. The slices were paraffin embedded and the sections, 3.20, 0.20 and $-2.80 \mathrm{~mm}$ (Scorza et al., 2005) of the bregma, were selected for the Nissl staining method. These sections were sectioned (Leica $^{\circledR}$ microtome, model RM2255), for obtaining cross-sections of $10 \mu \mathrm{m}$ thick. After deparaffinization and hydration in descending alcohol series, the sections were stained with cresyl violet solution (Sigma-Aldrich) at $0.5 \%$ for $30 \mathrm{~min}$, for the marking of Nissl bodies present in the cytoplasm of neurons. Then we proceeded to assemble and analyse the slides (Alvarez-Buylla et al., 1990; Scorza et al., 2005).

\section{Immunohistochemistry}

Sections of $3 \mu \mathrm{m}$ of the intervals of $3.20 \mathrm{~mm}, 0.20$ $\mathrm{mm}$ and $-2.80 \mathrm{~mm}$ to the bregma were deparaffinized in xylene under heating in an oven at $60^{\circ} \mathrm{C}$ for $30 \mathrm{~min}$. The primary antibody used was to GFAP (1:200, Anti-GFAP, Sigma-Aldrich). For antigen retrieval, a pre-treatment with citrate buffer $\left(\mathrm{pH} \mathrm{6.0)}\right.$ ) at $95^{\circ} \mathrm{C}$ was used for $30 \mathrm{~min}$ in the microwave. After washing with PBS a buffer containing $2 \%$ Triton X-100 + bovine serum albumin +
Tween 20 was used to block non-specific reactions and leave the more permeable fabric. Then, the sections were immersed in $3 \% \mathrm{H}_{2} \mathrm{O}_{2}$ to block endogenous peroxidase. Sections were incubated with primary antibody overnight in a humidified chamber at $4^{\circ} \mathrm{C}$. Then, the sections were washed in PBS and incubated with biotinylated secondary antibody (Dako LSABß2 system-HRP, DakoCytomation) for $2 \mathrm{~h}$ and then with streptavidin-peroxidase conjugate for $45 \mathrm{~min}$. For immunostaining the sections were revealed with chromogen solution of 3-3'diaminobenzidina tetrachloride (1:2; DAB, Sigma-Aldrich) and counterstained with hematoxylin. For the negative control, the histological sections did not receive the primary antibody.

\section{Morphometry}

For quantitative analysis of the cells three images of each histological section were captured, totaling nine images of each animal and 54 images for each group. To capture the images an Axio Vision Digital Camera (AxionCam ERC5S) coupled to a microscope (Zeiss Axio Lab A1), with $200 \times$ and an area of image analysis of $50 \times 10^{6} \mu \mathrm{m}^{2}$ (version 4.8 ), was utilized. Then, the cells of the selected images were quantified with the aid of the image J software program (Image-Pro Plus, version 4.5, Windows 98), yielding an average of cells of each histological section. Quantification was performed in both cerebral hemispheres of the specific regions of each section in relation to the bregma: In the $3.20 \mathrm{~mm}$ section two regions were considered: The edge of the motor cortex region and the penumbra area of the ischemia; in the $0.20 \mathrm{~mm}$ section the transition between the motor cortex and the striatum was considered; and in the -2.80 $\mathrm{mm}$ section the apex region of the dentate gyrus (hippocampus) was measured. For quantification of neurons, we considered the larger cells with nucleus limits and a well-defined nucleolus, as well as the marking of Nissl bodies in the cytoplasm of neurons (Scorza et al., 2005). For the quantification of GFAPimmunoreactive astrocytes, cells were considered in which cytoplasm showed brown coloration.

\section{Statistics}

The analysis of the mean values of the density of neurons and astrocytes, between the two groups were analyzed by one-way ANOVA test followed by posthoc Tukey, considering a significance level of $\mathrm{p}<0.05$. Results were expressed as mean \pm Standard Error of the Mean (SEM). 


\section{Results}

\section{Neurons}

The density of neurons in the apex of the dentate gyrus of the hippocampus of animals with cerebral ischemia was significantly different in the right $(p<0.05)$ and left $(p<0.05)$ brain hemispheres comparing the animals that exercised before MCAO with the sedentary group. In the control group no difference was observed in either of the cerebral hemispheres $(p>0.05)$.

The study identified a greater density of neurons in the striatum of the right cerebral hemisphere $(p<0.05)$ for the group that exercised before MCAO compared with the sedentary animals and those that exercised after MCAO. In the left hemisphere a greater density of neurons was also identified for the group exercised before MCAO compared with the untrained group $(p<0.05)$.

In the control group, there was a greater density of neurons in the striatum of the left cerebral hemisphere of the animals that exercised before surgery compared with those that exercised after surgery and that were sedentary $(p<0.05)$. In the right cerebral hemisphere no difference was observed between the groups $(p>0.05)$.

Analysis of the density of neurons in the penumbra area of the right cerebral hemisphere identified differences $(p<0.05)$ between the group exercised prior to MCAO and the sedentary group and also the group that was exercised after cerebral ischemia (Fig. 1C). In the left cerebral hemisphere no difference was observed between the groups (Fig. 1D).

After analysis of the density of neurons at the edge of the cerebral infarction in both cerebral hemispheres, no difference between the groups was observed. However, the corresponding areas in the motor cortex of the cerebral hemispheres of the control group had lower cell density in the group of animals that was exercised after surgery compared with the sedentary group $(p<0.05)$.

\section{Astrocytes}

Quantification of astrocytes in the apex of the dentate gyrus of the hippocampus of both hemispheres revealed no significant difference in the immunoreactivity of GFAP in astrocytes between the groups with cerebral ischemia. Quantification of astrocytes in the striatum of the right cerebral hemisphere showed no significant difference. In the left cerebral hemisphere the number of GFAP-reactive cells in the animals trained after cerebral ischemia was significantly different $(p<0.05)$ from that in the animals trained before the ischemia.
(A)

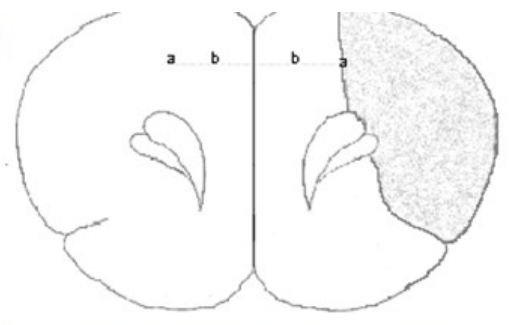

(B)

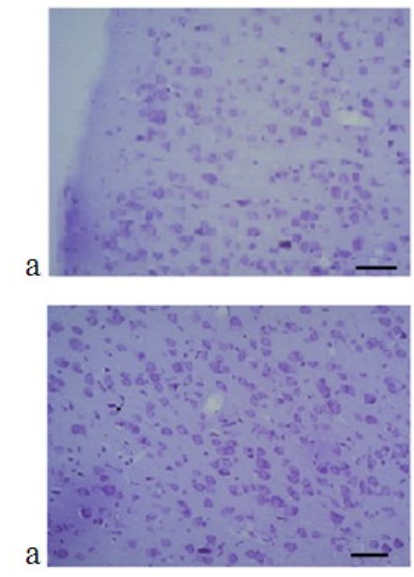

(C)
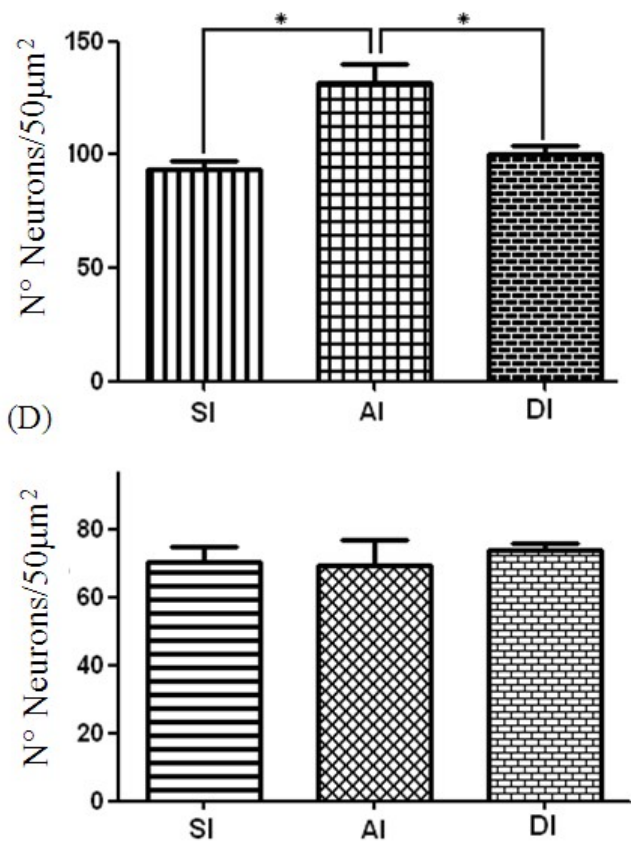

Fig. 1. Quantification of neurons in the motor cortex of the left and right cerebral hemispheres by the Nissl method. SI, sedentary group; AI, animals exercised prior to Middle Cerebral Artery Occlusion (MCAO); DI, animals that exercised after MCAO. (A) Scheme of the slice of the motor cortex, with representation from the edge of the infarct area and penumbra area of the motor cortex. (B) Neurons of the edge (a) and the penumbra area of the left cerebral hemisphere (b) of an AI group animal. $\mathrm{Bar}=50 \mu \mathrm{m}$. (C) Quantification data of neurons in the penumbra region of the right cerebral hemispheres. (D) Quantification data of neurons in the penumbra region of the left cerebral hemisphere. SI, sedentary group; DI, animals that exercised after MCAO. Mean $\pm \operatorname{SEM}(n=6 /$ group $) .{ }^{*} p<0.05$, by one-way ANOVA, followed by the post hoc Tukey test 
A greater number of astrocytes at the edge of the motor cortex of the left and right cerebral hemispheres were observed in the animals exercised before ischemia compared with the sedentary group $(p<0.05)$. In the penumbra area of the motor cortex of the right cerebral hemisphere there was a greater amount of astrocytes in animals trained before cerebral ischemia compared with the sedentary group $(p<0.05)$. In the penumbra area of the left cerebral hemisphere no difference was observed in the number of astrocytes in the groups with cerebral ischemia.

In the control group there was a significant increase in the number of astrocytes in the striatum and the motor cortex of the left and right cerebral hemispheres of animals that exercised before surgery compared with the sedentary animals and those exercised after surgery.

In the GFAP immunohistochemistry it is possible to observe the largest number of labeled astrocytes in the striatum and motor cortex of rats with cerebral ischemia of group AI in comparison with the SI group, in the left cerebral hemisphere. There was a greater number of reactive astrocytes for GFAP in the penumbra area of the right cerebral hemisphere in group AI compared with SI group animals. Equally, in the DI group, the presence of gemistocytic astrocytes (astrocytes with large cytoplasm) in the penumbra area of the right cerebral hemisphere.

\section{Discussion}

This study aimed to analyse the density of astrocytes and neurons in the brain of rats that performed exercise before or after ischaemia. The results indicated that the practice of physical exercise, before cerebral ischemia, benefited the morphology of brain tissue, with increased density of neurons in important regions of motor planning, such as the hippocampus, striatum and motor cortex.

The present study demonstrated that physical activity prior to the injury causes a decrease in neuronal death and consequent recovery in the injured area. In the study of Wang et al. (2001) the physical preconditioning increased angiogenesis by an increase in metabolic demand in cortical and subcortical regions of the rat brain. Endres et al. (2003) identified the increased expression of endothelial nitric oxide as a neuroprotective factor of physical activity in the rat brain with cerebral ischemia.

In the control group that exercised before surgery, an increase in neurons and astrocytes in the striatum and motor cortex of the cerebral hemispheres was also evident, which confirms the benefits of an exercise program for the prevention of brain disorders. There are several neurotrophic factors that are released as a result of physical training, allowing increased brain neuroplasticity (Kim et al., 2005).

The present study identified a greater density of neurons in the cerebral hemisphere contra lateral to the lesion in animals exercised prior to cerebral ischemia compared with sedentary animals. This can be justified by neuroplastic changes that occur in the region of the lesion (Chung et al., 2010; Kim et al., 2005), because after brain injury there is growth of axons in the contra lateral cortex that project to the motor cortex and striatum of the injured area, as a means of recovery of the impaired area (Carmichael and Chesselet, 2002; Jones et al., 2009). This process happens through the issuance of a neuronal stimulus of the area of injury for the neurons of the contralateral motor cortex region allowing cortical reorganization (Carmichael and Chesselet, 2002; Carmichael, 2003). Also involved in this process is the increased synthesis of neurotrophic factors such as brainderived neurotrophic factor and nerve growth factor in the motor cortex contralateral to the lesion (Chung et al., 2010, 2013; Kim et al., 2005; Vaynman et al., 2004).

In the present study of employee exercise program after cerebral ischemia there was no increase in the number of neurons in the brain areas analysed; however, there was an increase of astrocytes in the striatum of the hemisphere that suffered the lesion. These results are important because astrocytes perform various functions in the brain and the repair of the injured area is helped by the release of growth factors that stimulate fibroblasts to deposit proteins in an extracellular matrix for tissue remodeling of the lesion (Eddleston and Mucke, 1993). Even so, physical exercise should be considered beneficial since it improves muscle strength, motor performance, coordination and balance of animals (Ding et al., 2004a; Zhang et al., 2012a).

The maximum velocity employed in this study was obtained gradually to minimize the impact on the region of the brain injury. However, it is believed that the beginning of the exercise program may have been too late (fourth postoperative day). Some studies that started the exercise program 1 day after cerebral ischemia showed recovery in the region of cerebral ischemia (Liu et al., 2011; Zhang et al., 2012a; 2012b).

Analysis of the GFAP-reactive astrocytes in the hippocampus of the rats of the present study demonstrated a lower density of these cells at the apex of the dentate gyrus compared with the striatum and motor cortex, confirming the results of other studies where some particularities of hippocampal morphology were observed (Eddleston and Mucke, 1993; Ridet et al., 1997). Although the data of this study show a lower density of astrocytes in the hippocampus, the quantification of neurons showed that rats trained before cerebral ischemia showed a greater density of neurons compared with the sedentary group. These results can be supported by some studies that demonstrate the benefits of physical exercise in the rescue and recovery of neuronal death in the injured area (Altman and Das, 1965; Curlik and Shors, 2011; Curlik et al., 2013; Ploughman et al., 2007).

The highest density of astrocytes demonstrated in the motor cortex of rats trained before ischemia and in the striatum of rats trained after cerebral ischemia 
demonstrates the interference of physical exercise on the number of these cells and therefore their participation in the repair of the region of the brain injury in this study. Astrocytes are important in modulating neuroplasticity and the complexity and versatility of these cells to repair nerve tissue depend on the diversity of the repertoire of markers, receptors, expression of factors as well as the location and extent of lesions (Ridet et al., 1997).

The animals trained before cerebral ischemia also benefited from a higher number of astrocytes in the motor cortex of the cerebral hemispheres, which may have contributed to neuronal survival. Astrocytes act in the regeneration of the brain tissue and microvascular integrity, interfering in the basal lamina and decreasing cerebral edema in ischemia (Ding et al., 2004b; 2006). The neurotrophic factors synthesized by astrocytes also protect the central nervous system, because they participate in neuronal calcium homeostasis and reduce toxicity at the lesion site (Eddleston and Mucke, 1993).

The results of this study show that there are important neuroprotective mechanisms in the regeneration of areas of ischemic injury in previously trained rats, but the exact mechanisms involved in this process need to be studied in more depth. Furthermore, different physical training protocols may interfere in different ways with the morphology of the rat brain.

\section{Conclusion}

The animals that exercised before cerebral ischemia had a greater density of neurons in the striatum, at the apex of the dentate gyrus of the hippocampus and in the motor cortex contra lateral to the lesion, as well as more reactive astrocytes in the motor cortex of the cerebral hemispheres.

Animals exercised from the fourth day after cerebral ischemia did not demonstrate a greater density of neurons in the apex of the gyrus dentate of the hippocampus, striatum and motor cortex of the brains analysed.

\section{Acknowledgement}

The present study was supported by the Program in Cellular and Structural Biology, Federal University of Viçosa. We sincerely thank all the anonymous reviewers for their helpful suggestions on the quality improvement of our paper.

\section{Funding Information}

The study was funded by the Graduate Program in Cellular and Structural Biology at the Federal University of Viçosa, Brazil.

\section{Author's Contributions}

Lead author of the manuscript is Laila Cristina Moreira Damázio; Co-authors of the manuscript are Raphael Teixeira Rocha Melo and Maira Castro Lima;
Collaboration researchers: Helio Batista dos Santos, Rosy Iara Maciel Ribeiro de Azambuja, Natalia Ribeiro Alves, Betânia Souza Monteiro; Co-supervisors: José Antônio Natali and Ricardo Junqueira Del Carlo; Advisor: Izabel Regina dos Santos Costa Maldonado.

\section{Ethics}

All procedures were approved by the Ethics Committee on Animal Use, Federal University of Viçosa (protocol 32/2011).

\section{References}

Abbott, N.J., 2002. Astrocyte-endothelial interactions and blood-brain barrier permeability. J. Anat., 200: 629-38. DOI: 10.1046/j.1469-7580.2002.00064.x

Altman, J. and G.D. Das, 1965. Autoradiographic and histological evidence of postnatal hippocampal neurogenesis in rats. J. Comparat. Neurol. 1965: 319-35. DOI: 10.1002/cne.901240303

Alvarez-Buylla, A., C. Ling and J.R. Kirn, 1990. Cresyl violet: A red fluorescent Nissl stain. J. Neurosci. Meth., 33: 129-33. DOI: 10.1016/0165-0270(90)90016-9

Arthur, F.E., R.R. Shivers and P.D. Bowman, 1987. Astrocyte-mediated induction of tight junctions in brain capillary endothelium: An efficient in vitro model. Brain Res., 433: 155-9. PMID: 3676853

Bergami, M., S. Santi, E. Formaggio, C. Cagnoli and C. Verderio et al., 2008. Uptake and recycling of proBDNF for transmitter-induced secretion by cortical astrocytes. J. Cell. Biol., 2183: 213-21. DOI: $10.1083 /$ jcb.200806137

Cammer, W., F.A. Tansey and C.F. Brosnan, 1990. Reactive gliosis in the brains of Lewis rats with experimental allergic encephalomyelitis. J. Neuroimmunol., 27: 111-20.

DOI: 10.1016/0165-5728(90)90060-Z

Carmichael, S.T. and M.F. Chesselet, 2002. Synchronous neuronal activity is a signal for axonal sprouting after cortical lesions in the adult. J. Neurosci. Meth., 22: 6062-70. PMID: 12122067

Carmichael, S.T., 2003. Plasticity of cortical projections after stroke. Neuroscientist, 9: 64-75. DOI: $10.1177 / 1073858402239592$

Chung, J.Y., M.W. Kim, M.S. Bang and M. Kim, 2010. The effect of exercise on trkA in the contralateral hemisphere of the ischemic rat brain. Brain Res., 1353: 187-93. DOI: 10.1016/j.brainres.2010.06.057

Chung, J.Y., M.W. Kim, M.S. Bang and M. Kim, 2013. Increased expression of neurotrophin 4 following focal cerebral ischemia in adult rat brain with treadmill exercise. PLOS ONE, 8: 524-61. DOI: 10.1371/journal.pone.0052461 
Curlik, D.M. and T.J. Shors, 2011. Learning increases the survival of newborn neurons provided that learning is difficult to achieve and successful. J. Cognit. Neurosci., 23: 2159-70. DOI: 10.1162/jocn.2010.21597

Curlik II, D.M., L.Y. Maeng, P.R. Agarwal and T.J. Shors, 2013. Physical skill training increases the number of surviving new cells in the adult hippocampus. PLOS ONE, 8: 1-9. DOI: 10.1371/journal.pone.0055850

Ding, Y., J. Li, Q. Lai, J.A. Rafols and X. Luan et al., 2004a. Motor balance and coordination functional outcome in rat with cerebral artery occlusion training enhances transient middle. Neuroscience, 123: $667-74$

DOI: 10.1016/j.neuroscience.2003.08.031

Ding, Y., J. Li, X. Luan, Y.H. Ding and Q. Lai et al., 2004b. Exercise pre-conditioning reduces brain damage in ischemic rats that may be associated with regional angiogenesis and cellular overexpression of neurotrophin. Neuroscience,124: 583-91. DOI: 10.1016/j.neuroscience.2003.12.029

Ding, Y.H., Y.C. Ding, J. Li, D.A. Bessert and J.A. Rafols, 2006. Exercise pre-conditioning strengthens brain microvascular integrity in a rat stroke model. Neurol. Res., 28: 184-9. DOI: 10.1179/016164106X98053

Duarte, S.G., A.D. Campos and B.O. Colli, 2003. Functional evaluation of temporary focal cerebral ischemia. Arquivo de Neuro-psiquiatria, 61: 751-6. DOI: $10.1590 / \mathrm{S} 0004-282 X 2003000500009$

Eddleston, M. and L. Mucke, 1993. Molecular profile of reactive astrocytes; implications for their role in neurologic diseases. Neuroscience, 54: 15-36. DOI: 10.1016/0306-4522(93)90380-X

Endres, M., K. Gertz, U. Lindauer, J. Katchanov and J. Schultze et al., 2003. Mechanisms of stroke protection by physical activity. Annal. Neurol., 54: 582-90. DOI: 10.1002/ana.10722

Eng, L.F., R.S. Ghirnikar and Y.L. Lee, 2000. Glial fibrillary acidic protein: GFAP-thirty-one years. Neurochem. Res., 25: 1439-51.

DOI: 10.1023/A:1007677003387

Goudstein, G.W. and A.L. Betz, 1986. The blood brain barrier. Science, 255: 70-9.

Henderson, C.E., H.S. Phillips, R.A. Pollock, A.M. Davies and C. Lemeulle et al., 1994. GDNF: A potent survival factor for motoneurons present in peripheral nerve and muscle. Science, 266: 1062-4. DOI: $10.1126 /$ science. 7973664

Humm, J.L., D.A. Kozlowski, D.C. James, J.E. Gotts and T. Schallert, 1998. Use-dependent exacerbation of brain damage occurs during an early post-lesion vulnerable period. Brain Res., 783: 286-92. DOI: 10.1016/S0006-8993(97)01356-5

Jones, T.A., R.P. Allred, D.L. Adkins, J.E. Hsu and A. O'Bryant et al., 2009. Remodeling the brain with behavioral experience after stroke. Stroke, 40: 135-8. DOI: 10.1161/STROKEAHA.108.533653
Kim, M.W., M.S. Bang, T.R. Han, Y.J. Ko and B.W. Yoon et al., 2005. Exercise increased BDNF and trkB in the contralateral hemisphere of the ischemic rat brain. Brain Res. 1052: 16-21. DOI: $10.1016 /$ j.brainres.2005.05.070

Kondo, T., H. Kinouchi, M. Kawase and T. Yoshimoto, 1996. Astroglial cells inhibit the increasing permeability of brain endothelial cell monolayer following hypoxia/reoxygention. Neurosci. Lett., 208: 101-4. DOI: 10.1016/0304-3940(96)12555-6

Li, J., Ding, Y.H., J.A. Rafols, Q. Lai and Y. Ding et al., 2005. Increased astrocyte proliferation in rats after running exercise. Neurosci. Lett., 386: 160-4. DOI: 10.1016/j.neulet.2005.06.009

Lim, S.H., J. Lee, J.I. Lee, J.I. Lee and S. Im et al., 2008. The quantitative assessment of functional impairment and its correlation to infarct volume in rats with transient middle cerebral artery occlusion. Brain Res., 1230: 303-9.

DOI: 10.1016/j.brainres.2008.07.002

Lin, F.H., D.H. Doherty, J.D. Lile, S. Bektesh and F. Collins, 1993. A glial cell line-derived neurotrophic factor for midbrain dopaminergic neurons. Science, 260: 1130-2. DOI: $10.1126 /$ science. 8493557

Liu, N., H. Huang, F. Lin, A. Chen and Y. Zhang et al., 2011. Effects of treadmill exercise on the expression of netrin-1 and its receptors in rat brain after cerebral ischemia. Neuroscience, 37: 45-55. DOI: 10.1016/j.neuroscience.2011.07.037

Longa, E.Z., P.R. Weinstein, S. Carlson and R. Cummins, 1989. Reversible middle cerebral-artery occlusion without craniectomy in rats. Stroke, 20: 84-91. DOI: 10.1161/01.STR.20.1.84

Ma, J., L. Zhao and J.R. Nowak, 2006. Selective, reversible occlusion of the middle cerebral artery in rats by an intraluminal approach optimized filament design and methodology. J. Neurosci. Meth., 156: 76-83.

DOI: $10.1016 /$ j.jneumeth.2006.02.006

Menzies, S.A., R.R. Smith, J.T. Hoff and A.L. Betz, 1992. Middle cerebral-artery occlusion in rats-a neurological and pathological evaluation of reproducible model. Neurosurgery, 31: 100-7. DOI: 10.1097/00006123-199207000-00014

Modo, M., R.P. Stroemer, E. Tang, T. Veizovic and P. Sowniski et al., 2000. Neurological sequelae and long-term behavioural assessment of rats with transient middle cerebral artery occlusion. J. Neurosci. Meth., 194: 99-109. DOI: 10.1016/S0165-0270(00)00329-0

Ploughman, M., S.G. Button, G. Chernenko, Z. Attwood and B.A. Tucher et al., 2007. Exercise intensity influences the temporal profile of growth factors involved in neuronal plasticity following focal ischemia. Brain Res., 1150: 207-16. DOI: 10.1016/j.brainres.2007.02.065 
Ridet, J.L., S.K. Malhotra, A. Privat and F.H. Gage, 1997. Reactive astrocytes: Cellular and molecular cues to biological function. Trends Neurosci., 20: 570-7. DOI: 10.1016/S0166-2236(97)01139-9

Scorza, F.A., R.M. Arida, R.M. Cysneiros, C.A. Scorza and M. Albuquerque et al., 2005. Estudo qualitativo da formação hipocampal de animais hipertensos com epilepsia. Arquivos de NeuroPsiquiatria, 63: 283-8. DOI: $10.1590 / \mathrm{S} 0004-282 X 2005000200015$

Senna, P.N., J. Ilha, P.P.A. Baptista, O.S. Nascimento and M.C. Leite et al., 2011. Effects of physical exercise on spatial memory and astroglial alterations in the hippocampus of diabetic rats. Metabolic Brain Disease, 4: 269-79.

DOI: $10.1007 / \mathrm{s} 11011-011-9262-\mathrm{x}$

Tao, C.J., Z. Nagy and M.W. Brightman, 1987. Tight junctions of brain endothelium in vitro are enhanced by astroglia. J. Neurosci., 7: 3293-9. PMID: 3668629

Taskapiliogly, M.O., T. Alkan, B. Goren, K. Tureyen and S. Sahin et al., 2009. Neuronal protective effects of focal ischemic pre-and/or postconditioning on the model of transient focal cerebral ischemia in rats. J. Clin. Neurosci., 16: 693-7. DOI: 10.1016/j.jocn.2008.07.077
Vaynman, S., Z. Ying and F. Gomez-Pinilla, 2004. Hippocampal BNDF mediates the efficacy of exercise on synaptic plasticity and cognition. Eur. J. Neurosci., 20: 2580-90. DOI: $10.1111 /$ j.1460-9568.2004.03720.x

Wang, R.Y., Y.R. Yang and S.M. Yu, 2001. Protective effects of treadmill training on infarction in rats. Brain Res., 922: 140-3. DOI: $10.1016 / \mathrm{S} 0006-8993(01) 03154-7$

Willis, C.L., C.C. Nolan, S.N. Reith, T. Lister and M.J.W. Prior et al., 2004. Focal astrocyte loss is followed by microvascular damage, with subsequent repair of the blood-brain barrier in the apparent absence of direct astrocytic contact. Glia, 45: 325-37. DOI: 10.1002/glia.10333

Zhang, P., Q, Zhang, H. Pu, Y. Wu and Y. Bai et al., 2012a. Very early-initiated physical rehabilitation protects against ischemic brain injury. Frontiers Biosci., 4: 2476-89. PMID: 22652654

Zhang, Q., Y. Wu, P. Zhang, H. Sha and J. Jia et al., 2012 b. Exercise induces mitochondrial biogenesis after brain ischemia in rats. Neuroscience, 205: 10-17. DOI: 10.1016/j.neuroscience.2011.12.053 